absolute limits of temporal resolution in the auditory pathway because it coincides with the shortest boundary of voice onset time perception in man, monkey, and chinchilla and with thresholds of perception of temporal order in humans (compare Ehret 1987). These cases show that categorical perception in Massaro's sense is likely to exist and should not be abandoned as a possibility for human speech unless falsified by physiological evidence. Finally, one has to consider the efferent auditory system that can influence tone discrimination even at the cochlear level and may be expected to influence speech processing as well (e.g. Winslow \& Sachs 1988). Thus top-down conditioning of the ascending auditory pathway is present and one might hypothesize that whenever categorical information in terms of speech phonemes is expected in a communicative context, peripheral processing is conditioned to accentuate these natural categories in order to preserve the semantic contents of the message optimally. If this is true, the categorization of speech information could gradually emerge in the auditory system and would hence be an inherent property of the perceptual process.

\section{A general algorithm for pattern recognition?}

\section{Gerd Gigerenzer}

Department of Psychology, Universität Konstanz, 7750 Konstanz, West Germany

\section{Electronlc mall: sygiger@dknkurz1.bitnet}

How does the brain make sense of the world? "In the same way that scientists do, and with the same tools," answer an increasing number of cognitive psychologists. The eighteenth-century mathematicians Laplace and Condorcet used their "probability of causes" to model the way scientists reason (Daston 1988); Dominic Massaro now proposes the identical formula as an algorithm for pattern recognition in general and speech perception in particular. To show the generality of the algorithm (the "fuzzy logical model of perception (FLMP]") is the ambitious goal of Massaro's book: "well-learned patterns are recognized in accordance with a general algorithm, regardless of the modality or particular nature of the patterns" (p. 16). The project of reducing pattern recognition to any algorithm, much less a single one, may strike many as overly ambitious. For the purposes of this commentary, however, I will accept Massaro's goal as in principle attainable and will try to invite him to clarify one of his major arguments by revealing its conceptual difficulties.

This argument is central to the issue of generality, and runs like this: (i) The FLMP is not only general but also optimal since it is mathematically equivalent to Bayes' theorem; (ii) here Bayes' theorem is implemented as it was by Laplace, that is, with the assumption of uniform prior probabilities and independence of events (features), but (iii) this equivalence poses a dilemma for the FLMP, since previous research, in particular on intuitive probabilistic reasoning, has rejected Bayesian reasoning as a general mental algorithm. In analyzing this argument, I shall proceed from the general to the specific.

1. Is the general algorithm a Bayesian one? The FLMP assumes that pattern recognition occurs in three sequential stages. I shall consider the simplest case, with only two features and two prototypes. In the feature evaluation stage, the match $t\left(E_{1} / H_{1}\right)$ between a feature $E_{1}$ and a prototype $H_{1}$ is calculated; in the feature integration stage the overall match $t\left(E / H_{1}\right)=$ $t\left(E_{1} / H_{1}\right) t\left(E_{2} / H_{1}\right)$ between the two features and the prototype $H_{1}$ is calculated; and in the pattern classification stage the probability that the pattern will be identified as $H_{1}$ is given by $p\left(H_{1} / E\right)$
$=t\left(E / H_{1}\right) /\left(t\left(E / H_{1}\right)+t\left(E / H_{2}\right)\right)$. Massaro (pp. 196-98) says that this algorithm is mathematically equivalent to Bayes' theorem, assuming uniform prior probabilities and independent events, and replaces the above $t$ (i.e. truth) values by $p$ (i.e. probability) values. However, Massaro repeatedly (e.g. pp. 21, 166, 202) asserts that $t\left(E_{1} / H_{1}\right)+t\left(E_{1} / H_{2}\right)=1$, which is not true for the corresponding probabilities $p\left(E_{1} / H_{1}\right)$ and $p\left(E_{1} / H_{2}\right)$ in Bayes theorem. According to standard probability theory, which mathematically implies Bayes' theorem, the sum of these probabilities can be either less or more than 1 . Thus, I doubt that the proposed general algorithm is in fact mathematically equivalent to Bayesian probabilities, and I therefore also doubt the claim that "either of these two models is adequate to account for the results" (p. 198).

2. A general pattern recognitlon algorithm with uniform priors? To keep the next points separate from the first, let me assume that I have overlooked something and that Massaro is right in pointing to the equivalence of the FLMP and Bayes' theorem. Laplace's urn analogy and Bayes' billiard table suggested uniform prior probabilities on the grounds that our ignorance gives us no reason to expect one urn or one area on the table to be a priori more likely than any other. But should we assume that a general pattern recognition algorithm also works on the principle of ignorance and uses uniform priors? An algorithm with uniform priors may be sufficient for the experimental designs reported in the book, in which the prototypes to be identified, such as $/ \mathrm{ba} /$ and $/ \mathrm{da} /$, are equally likely in the laboratory. But in everyday speech, just as in many other domains, different patterns have different prior probabilities depending on context. Where expectation plays a role, nonuniform priors seem to be indispensable for improving the perceptual "bet" in situations with uncertain information. ${ }^{1}$

3. Does intuitive probabilistic reasoning challenge the generality of the algorlthm? Massaro argues that the mathematical equivalence of the FLMP and Bayes' theorem "poses a new dilemma" for the generality claim, since previous research has rejected Bayes' theorem as a model of intuitive probabilistic reasoning. His major defense is that most previous researchers used "objective" rather than "subjective" probabilities to calculate the so-called normative Bayesian outcome. Massaro's reply is correct: There are many ways to be a Bayesian, and such experiments do not rule out that intuitive reasoning is Bayesian by using subjective probabilities. But there are designs, such as in Kahneman and Tversky's (1973) Engineer-Lawyer study, which allow for subjective likelihoods and which still lead the authors to conclude that reasoning is not Bayesian, since base rates are ignored due to a representativeness heuristic. Massaro has to deal with these kinds of experiments. Moreover, even in the Engineer-Lawyer Problem, the neglect of base rates can easily be eliminated if one crucial structural assumption (random sampling of description) is made vivid to subjects, although the feature values remain constant (Gigerenzer et al. 1988). Such systematic changes in reasoning indicate that neither representativeness (i.e. uniform-prior Bayesianism, see below) nor Bayes' theorem is a general algorithm of the mind. Massaro's "dilemma," in my opinion, is not that intuitive reasoning ignores base rates (as does the FLMP in the book under review), but rather that intuition seems to have a whole toolbox of algorithms available.

Two things puzzle me concerning the relation between reasoning and the FLMP. First, as noted above, Massaro says that the pattern recognition algorithm is a Laplacean uniform-prior variant of Bayes' theorem. Why then does he believe it is a "dilemma" that intuitive reasoning seems to violate Bayesian reasoning by neglecting base rates and using uniform priors? Base rate neglect is exactly what his algorithm predicts - just as Baconian probability would (Cohen 1986). [See also Cohen: "Can Human Irrationality Be Experimentally Demonstrated?" BBS 4(3) 1981 and Kyburg: "Rational Belief" BBS 6(2) 1983.] 
Second, what is the relationship between Kahneman and Tversky's representativeness heuristic and the FLMP as models of pattern recognition? Massaro says they are fundamentally different (p. 273). However, since in this context "representativeness" can be shown to mean Bayesian reasoning with uniform priors (Gigerenzer \& Murray 1987, Chapter 5), I understand both the FLMP and the heuristic to refer to the same strategy - although the FLMP is a model and representativeness is just a word.

4. The "conjunction fallacy" and the FLMP. What has been called a "conjunction fallacy" is a judgment of the following kind: A fictitious person named Linda is more likely to be a feminist and a bank teller than just a bank teller. I agree with Massaro that this cannot be called a "fallacy" unless one willfully ignores the fact that the term "likely" has several meanings in everyday language. But I part ways with him when he extends the generality of the FLMP to conjunction judgments and claims that "within Bayes theorem or the FLMP, we can predict that Linda will be rated as being more likely [to be] a feminist and bank teller than just a bank teller" (p. 275). Massaro's argument is that the subjects behave as if they were carrying out pattern recognition, evaluating the features against alternative prototypes. In fact, the FLMP predicts that the probability that a pattern is recognized as prototype $H_{1}$ can be larger for two features $E_{1}$ and $E_{2}$ than for just $E_{1}$ alone. In formal terms, this means that $p\left(H_{1} / E_{1} \& E_{2}\right)>p\left(H_{1} / E_{1}\right)$. But this is not the "conjunction fallacy," which is to judge $p\left(H_{1} \& H_{2} / E_{1}\right)>p\left(H_{1} / E_{1}\right)$. Only the latter contradicts standard probability theory, and thus cannot be derived from either Bayes' theorem, which is a consequence of standard probability theory, or the FLMP, insofar as it is claimed to be mathematically identical to the former. ${ }^{2}$

5. Why only one algorlthm? Scientific reasoning is a manysplendored thing, encompassing many and diverse forms of inference. We might want to carry the analogy between scientific reasoning and cognition far enough to extend this multiplicity to the mind. Thus, instead of one general, all-purpose algorithm, we would expect to find several or even many algorithms. There is an additional Darwinian argument against the view that evaluation has given us only a single algorithm for all cases of pattern recognition. Since each algorithm assumes a specific structure (e.g. in Massaro's model, independence of features and a mutually exclusive and exhaustive set of prototypes), it is well equipped for specific tasks that have these structures, but less so for other tasks. In order to survive in a changing environment, the mind would be better off if outfitted with a whole toolbox of algorithms, and with an evaluation program that first checks the structure of the environment before it selects a particular algorithm to apply to that environment.

I have concentrated in this commentary on conceptual issues that need further clarification. These have to do with the claims for a general algorithm for pattern recognition, but do not touch the valuable experimental work that the book presents, which I have not here addressed.

\section{NOTES}

1. However, nonuniform priors may not be required to improve the fit of the FLMP to experimental data, since it is already excellent. My point here is a conceptual one. It is in fact hard to judge to what degree the FLMP is supported by the empirical data, because of the large number of free parameters (sometimes close to $50 \%$ of the data points) that can be fitted to the data. The true degree of support could be revealed by a step-wise cross-validation procedure: Use the best-fitting feature values for given prototypes and a given subject in a new experiment with the same or an enlarged set of features. If features are evaluated independently from other features present, as the FLMP proposes, then the feature values should be stable, and the fit in the second experiment would provide a stronger test for the validity of the FLMP.
2. In his own experiments, Massaro (submitted) showed that the FLMP gives an excellent fit to judgments in Linda-type tasks. However, judgments of the type $p$ (Linda/vocation\&avocation) were compared with $p$ (Linda/vocation) and $p($ Linda/avocation $)$, which, as mentioned above, cannot violate the conjunction rule.

\section{From speech perception to person perception? Not quite yet}

\section{Sam Glucksberg}

Department of Psychology, Princeton University, Princeton, NJ 08544 Electronic mail: samg@pucc.bitnet

Massaro's basic argument is that people integrate information from multiple sources in order to decide among alternatives, whether those alternatives are two syllables, or two types of people. Massaro's strong claim is that the specific domain of alternatives is irrelevant: The processes that are used to perceive speech sounds are also used to perceive people, or to interpret sentences, or to learn and use natural categories. The specific processing model is not at issue here. Instead, I want to examine two claims. The first claim is that the theoretical conclusions concerning bimodal speech perception generalize to quite disparate domains, including such domains as person perception and sentence interpretation. The second claim is that the findings and the overall theoretical approach are incompatible with modularity on the one hand, but compatible with connectionism on the other hand.

With respect to the first claim, at some level it must be true. People must integrate information from various sources irrespective of stimulus domain - providing, of course, that more than one information source is available. Because such variables as the intensity and frequency of an auditory stimulus are considered to be two information sources, virtually all percepts and judgments would perforce depend upon multiple information sources. The generality claim, however, is stronger than this. Person perception and sentence interpretation, among others, "might follow the algorithm of the FLMP [fuzzy logical model of perception] that has proven appropriate for speech perception" (Massaro 1987, p. 245). This is an interesting hypothesis, but I seriously question the choice of experimental paradigms for evaluating it.

For the domain of person perception, Massaro relies on an experimental paradigm developed by Anderson (1973) and his colleagues (e.g., Birnbaum 1974). People are given two or more adjectives in every possible combination, and for each combination rate some person-characteristic. Ratings of social desirability, likableness, and introversion are examined and found to be consistent with model predictions. Massaro concludes that person perception is accomplished the same way that speech perception is: by integrating multiple, continuous, and independent stimulus attributes. The data and model fits are consistent with Massaro's claim. But do these data reflect person perception processes, or is it more likely that they reflect the demands of the experimental task?

Massaro reports a typical experiment on "person perception." College students rated how introverted or extraverted an unspecified, hypothetical person was on the basis of either a single descriptor, such as entertaining, or a pair of descriptors, such as entertaining and withdrawn. Each student rated each of eight such adjectives one at a time, and then rated all 16 pairs, one pair at a time. The results were in accord with Massaro's FLMP. Each adjective contributed independently to the judgments, leading Massaro to conclude that the contribution of each adjective was independent of the other adjective, Asch's classic demonstration (1946) of meaning interaction in person impression notwithstanding.

It may be misleading to interpret such studies as reflecting person perception processes, however. To begin with, there are 